\title{
Book Review: Role of Biofilms in Bioremediation
}

\author{
Santosh Kr. Karn ${ }^{1,2 *}$, Jizhou Duan ${ }^{1}$ and lan R. Jenkinson ${ }^{3,4}$ \\ ${ }^{1}$ Key Laboratory of Environmental Corrosion and Biofouling, Institute of Oceanology, Chinese Academy of Sciences, \\ Qingdao, China, ${ }^{2}$ Department of Biotechnology, National Institute of Technology, Riapur, India, ${ }^{3}$ Key Laboratory of Marine \\ Ecology and Environmental Pollution, Institute of Oceanology, Chinese Academy of Science, Qingdao, China, ${ }^{4}$ Agency for \\ Consultation and Research in Oceanography, Lavergne, France
}

Keywords: biofilm, bioremediation, microbe, sustainable, technology

\section{A Book Review on \\ Biofilms in Bioremediation: Current Research and Emerging Technologies}

Gavin Lear (Caister: Academic Press), 2016, 252 pages, ISBN: 978-1-910190-29-6 and cover page given in Figure 1.

This book treats the roles of biofilms in bioremediation. Biofilms, here taken to include flocs, comprise physical structures that support ecosystems of microorganisms. In turn, the microorganisms construct and engineer these biofilms, forming microbial communities (Flemming and Wingender, 2010). Biofilms are organized as stable communities of mono- or multi-specific cells of bacteria with or without eukaryotes, attached to each other and surfaces in different environments (Costerton, 1995). In the last few years, many novel, organic compounds have been developed, and are released into the environment. They include heavy metals, polyaromatic hydrocarbons, polychlorinated biphenyls, pesticides, chemical fertilizers, detergents, paints, disinfectants, lubricants, antibiotics, and nanoparticles. Many of them are toxic to humans and other organisms. Managing the effects of these pollutants is a challenge to sustainable development globally. Using biofilms as a tool in bioremediation of such potentially harmful affects can allow new technologies to remain environmentally sustainable if integrated methods of use are correctly developed and applied. Such technology, using microorganisms and their products to degrade and clean up a large variety of pollutants are advantageous, and they need to be integrated into protocols that minimize: disruption of land or wildlife in the treated region; noise and dust; release of harsh chemicals. Numerous studies have been made on how biofilms relate to disease and industries, but less focus has been given to the biofilms related to the environment. One striking example, however, relates to current cleaning of the major oil spill by Exxon Oil on the shorelines of Prince William Sound, Alaska, back in 1989 (Bragg et al., 1994; Atlas and Hazen, 2011). Consortia of genetically modified microbes have been deployed to detoxify pollutants. Such biofilm research in the natural environment of soil, sand, and sediments has revealed the potential of biofilms in treating pollutants.

The book consists of 13 chapters providing up-to-date information on biofilms in bioremediation of different environmental pollutants. Chapters 2, 3, and 4 explain the basic biofilm concepts particularly as related to: (1) bioremediation; (2) biofilm survival strategies through changes in their extracellular matrix when challenged by pollutants; (3) cellular biochemistry; (4) cellular social interactions; (5) exchange of metabolites; (6) signaling molecules; (7) genetic control of chemotactic responses by bacteria to pollutants. Chapters 1, 8, and 10 describe approaches for successful bioremediation, highlighting polycyclic aromatic hydrocarbons (PAH), pesticides and 


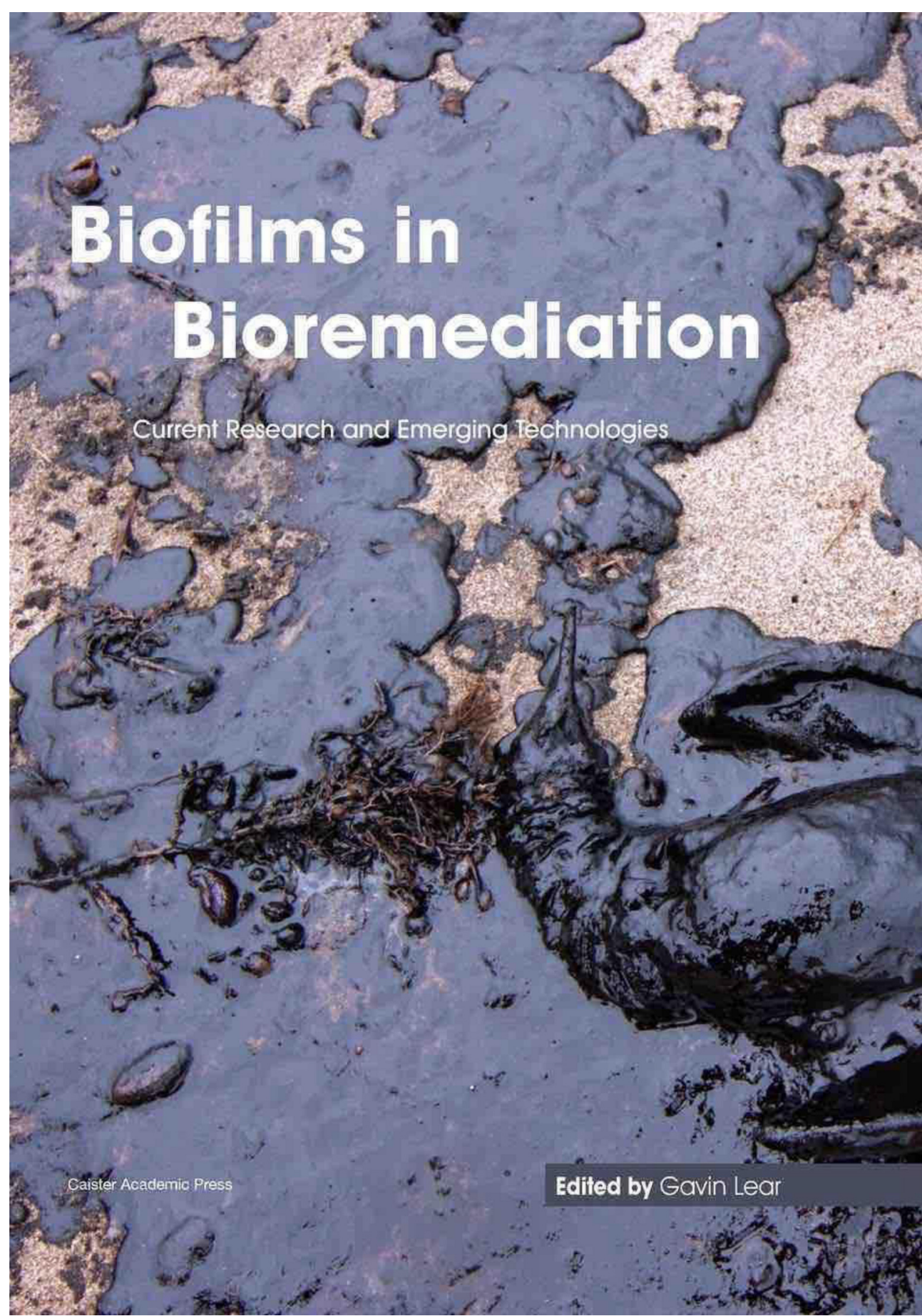

FIGURE 1 | Book covering page.

hydrocarbon degradation. Chapter 9 describes the removal of Chromium (VI) from industrial wastewater using a bacterial biofilm. Chapters 6 and 7 describe microscopic and molecular methods for monitoring bioremediation. Chapter 5 treats biosensors applied to detect bioavailability of contaminants and pollutants. Chapter 11 describes the engineering of permeable reactive barriers $(\mathrm{PRB})$ within contaminated aquifers to manage migration of mobile pollutants. Chapter 12 investigates the formation of Pseudoalteromonas bacterial biofilm in fish farms These biofilms produce extracellular proteases, which remove excess proteinaceous material from sediment and sludge, thus maintaining good water quality. How biofilm-associated cells 
of Pseudoalteromonas stutzeri promote naphthalene degradation is also explained. Chapter $13 \mathrm{~A}$ deals with the potential role of microbial biofilm in enhancing phytoremediation linked to phenol degradation, and further explain the role of microbial biofilm in development of duckweed populations. Chapter 13B describes how microbial biofilms are applied to enhance phytoremediation of contaminants in soil and water through Phragmites australis rhizospheres.

While the book describes well the basic principles of biofilm in the degradation of organic pollutants, there would have been room for more elaboration about removal of pollutants and manipulation of biofilms for bioremediation under different condition. In the place of Chapter 12, for example, it would have been good to see inclusion of molecular approaches and analysis of biofilms in soil water. Commonly these microbial biofilms work in natural environments, but some modifications can promote microbial degradation of pollutant more rapidly. Better understanding of microbial biochemistry, physiology, and genetics will help further develop microbial processes to focus bioremediation better on particular functional problems. New post-genomics technologies such as metabolomics represent a rapidly emerging field that is likely to enhance understanding of biological mechanisms. Metabolomics applied in bioremediation studies, will help elucidate mineralization process, while pathways using isotope distribution analysis, molecular connectivity analysis, and metabolic foot-printing analysis, may all improve targeted biodegradation processes using metabolic engineering. In short, the field of bioremediation has much to gain from this emerging area.

\section{REFERENCES}

Atlas, R. M., and Hazen, T. C. (2011). Oil biodegradation and bioremediation: a tale of the two worst spills in U.S. history. Environ. Sci. Technol. 45, 6709-6715. doi: 10.1021/es2013227

Bragg, J. R., Prince, R. C., and Harner, E. J. (1994). Effectiveness of bioremediation for the Exxon Valdez oil spill. Nature 368, 413-418.

Costerton, J. W. (1995). Overview of microbial biofilms. J. Ind. Microbiol. 15, 137-140.

Flemming, H.-C., and Wingender, J. (2010). The biofilm matrix. Nature 8, 623-633. doi: $10.1038 /$ nrmicro 2415
In short, this book clearly explains the potential roles of biofilms in bioremediating soils, sediments, and aquifers containing both organic and inorganic pollutants. It also brings together descriptions of recent tools and information about microbial biofilms applied to degradation and phytoremediation of industrial waste. In addition, it describes the significance of permeable, reactive barriers for the treatment of mobile pollutants. The book will help the microbiologist, the biotechnologist, and the environmental engineer to develop and integrate more sustainable technologies for bioremediation of industrial wastes. It will be useful or even indispensable to teachers, students, and researchers in the life sciences, particularly of industrial biotechnology and environmental microbiology.

\section{AUTHOR CONTRIBUTIONS}

SK is the primary author and initiator of this book review, while JD and IJ have both contributed to writing this review at SK's request.

\section{ACKNOWLEDGMENTS}

This work was supported by the Program of Visiting Research Scientist of the Chinese Academy of Sciences (Grant No. Y3KY02013L) and PIFI project of Chinese Academy of Science (Grant No. 2016VBC077). Authors are also thankful to Key Laboratory of Environmental Corrosion and Biofouling, Institute of Oceanology, Chinese Academy of Science Qingdao, China for providing facility, space, and resources for this work.

Conflict of Interest Statement: The authors declare that the research was conducted in the absence of any commercial or financial relationships that could be construed as a potential conflict of interest.

Copyright (c) 2017 Karn, Duan and Jenkinson. This is an open-access article distributed under the terms of the Creative Commons Attribution License (CC $B Y)$. The use, distribution or reproduction in other forums is permitted, provided the original author(s) or licensor are credited and that the original publication in this journal is cited, in accordance with accepted academic practice. No use, distribution or reproduction is permitted which does not comply with these terms. 